\title{
Abundância e sazonalidade de Bothriurus signatus (Arachnida, Scorpiones) em diferentes formações vegetais em São Francisco de Paula, RS, Brasil
}

\author{
Ricardo $\mathrm{Ott}^{1} \&$ Ana Paula Ott ${ }^{2}$
}

1. Museu de Ciências Naturais, Fundação Zoobotânica do Rio Grande do Sul, Rua Dr. Salvador França, 1427, 90690-000, Porto Alegre, RS, Brasil. (rott@fzb.rs.gov.br).

2. Departamento de Fitossanidade, Faculdade de Agronomia, Universidade Federal do Rio Grande do Sul, Av. Bento Gonçalves, 7712, 91540-000, Porto Alegre, RS, Brasil. (ana.ott@ufgrs.br).

\begin{abstract}
Abundance and seasonality of Bothriurus signatus (Arachnida, Scorpiones) in different vegetal formations in São Francisco de Paula, RS, Brazil. A survey of litter scorpions was conducted in three types vegetation (primary forest, secondary forest and silvicultures) in an area of Araucaria forest in southern Brazil. Pitfall traps were used over 20 months for the sampling of scorpions. A total 165 scorpions of Bothriurus signatus Pocock, 1893 were sampled. Males were more abundant than females and these more abundant than juveniles. The scorpions were more abundant in primary forest and silvicultures than in secondary forest. The activitie peak of scorpions was registered during the spring. The total length of the adult scorpions ranged from 3.05 to $4.52 \mathrm{~cm}$ with males significantly larger than females. No pregnant females were registered among collected specimens.
\end{abstract}

KEYWORDS. Seasonality, Neotropical, Scorpions, Araucaria Forest.

RESUMO. Foi realizado levantamento de escorpiões em serrapilheira de três tipos de formações vegetais (florestas primárias, florestas secundárias e silviculturas) em área de Floresta com Araucária no Sul do Brasil. Para a coleta foram utilizadas armadilhas de queda ao longo de 20 meses. No total foram amostrados 165 escorpiões de apenas uma espécie identificada como Bothriurus signatus Pocock, 1893. Machos foram mais abundantes que fêmeas e jovens. A espécie foi mais abundante em áreas de floresta primária e silvicultura do que na floresta secundária. A maior atividade dos escorpiões foi registrada durante a primavera. O comprimento total dos escorpiões adultos coletados variou de 3,05 a 4,52 cm sendo que machos foram significativamente maiores que fêmeas. Nenhuma fêmea grávida foi registrada entre os indivíduos coletados.

PALAVRAS-CHAVE. Sazonalidade, Neotropical, escorpiões, Floresta com Araucária.

Os escorpiões estão entre as maiores ordens de aracnídeos, incluindo cerca de 1.500 espécies das quais cerca de 130 são conhecidas para o Brasil (BonaLDo et al., 2009; Brazil \& PoRTo, 2010). São considerados importantes elementos de estudos ecológicos, podendo ser afetados por uma série de fatores limitantes como temperatura, precipitação, características do solo e rochas, coberturas de serrapilheira e pedras, topografia, vegetação e fisiologia do ambiente (Polis, 1990; PrendinI, 2005).

Polis (1990) afirmou que a diversidade de escorpiões é em geral maior em áreas subtropicais entre as latitudes 23-38 , decrescendo em direção aos polos e ao Equador. Segundo o mesmo autor, em áreas propícias à ocorrência de escorpiões é possível encontrar até 13 espécies em uma comunidade local; todavia, em $68 \%$ dos locais com estudos sobre a diversidade da fauna de escorpiões são registradas entre três e sete espécies simpátricas.

Dados sobre a diversidade de assembleias de escorpiões no sul do Brasil são inexistentes, contudo, estudos realizados no Uruguai confirmaram o registro de até quatro espécies nativas simpátricas (ToscanoGADEA, 2002). De acordo com a coleção de escorpiões do Museu de Ciências Naturais da Fundação Zoobotânica do Rio Grande do Sul, seis espécies são registradas no estado do Rio Grande do Sul: Bothriurus bonariensis Koch, 1842; Bothriurus signatus Pocock, 1893; Tityus costatus Karsch, 1879; Tityus uruguayensis Borelli, 1901; Urophonius iheringi Pocock, 1893 e Zabius gaucho Acosta et al., 2008 (R. Ott, dados inéditos). Em outras regiões do Brasil o número de espécies simpátricas de escorpiões pode chegar até seis, dependendo da região e dos microambientes amostrados (Dias et al., 2006; Bonaldo et al., 2009; AraúJo et al., 2010).

O objetivo deste estudo é conhecer a abundância e a sazonalidade de $B$. signatus em serrapilheira de floresta primária, floresta secundária e silvicultura de Pinus spp. no município de São Francisco de Paula, RS, ao longo de 20 meses de amostragem. Adicionalmente foram realizadas medidas do tamanho corporal dos exemplares para verificar possíveis variações entre os sexos e entre os escorpiões das diferentes formações vegetais, assim como dissecação de fêmeas para verificação da presença de embriões.

\section{MATERIAL E MÉTODOS}

O estudo foi realizado no Centro de Pesquisas e Conservação da Natureza Pró-Mata (CPCN Pró-Mata), em São Francisco de Paula, Rio Grande do Sul, Brasil $\left(29^{\circ} 27^{\prime}-29^{\circ} 35^{\prime} \mathrm{S}, 50^{\circ} 08^{\prime}-50^{\circ} 15^{\prime} \mathrm{O}\right)$. A temperatura média anual é de $14,5^{\circ} \mathrm{C}$ e a precipitação anual é de $2.252 \mathrm{~mm}$ (Bertoletti \& Teixeira, 1995). O clima é mesotérmico superúmido, com chuvas bem distribuídas ao longo do ano sem período seco e do tipo "Cfbl", subtropical segundo a classificação de KöPPEN (1948), com a média das máximas inferior a $22,9^{\circ} \mathrm{C}$ e a média das mínimas $12,3^{\circ} \mathrm{C}$ (BACKeS, 2009). Valores médios de pluviosidade e temperatura para 
o período foram obtidos a partir dos dados da EMBRAPA Uva e Vinho, Bento Gonçalves, RS e ajustados para São Francisco de Paula através da aplicação de índices mensais baseados nas médias dos anos de 1930 a 1960 (МотA \& Agendes, 1986).

Caracterização da área de estudo e formações vegetais amostradas. A área localiza-se no extremo leste do Planalto Sul-brasileiro, com altitudes entre 600 e $950 \mathrm{~m}$. A topografia é composta principalmente de áreas escarpadas da encosta da Serra Geral sendo que na sua porção noroeste localiza-se uma área com características típicas do Planalto Sul-rio-grandense (BAASKE, 2001). A vegetação constitui-se de um misto de campos sulinos e florestas com Araucária (Floresta Ombrófila Mista) nas áreas do planalto e no topo das escarpas; nas encostas, até o limite superior de cerca de $900 \mathrm{~m}$, podem ser encontradas florestas típicas da Costa Atlântica Brasileira (Floresta Ombrófila Densa) (BAASKE, 2001). Segundo BAASKE (2001) toda a área do CPCN PróMata sofreu impacto da ação humana, com criação de gado e cortes seletivos de madeira nas diferentes formações vegetais, desde 1950. A partir de 1980 a antropização decaiu e foi definitivamente interrompida em meados da década de 1990, quando ocorreu a transformação do local em um centro de pesquisas.

As amostragens foram realizadas em três formações vegetais distintas: mata primária com presença de pinheirobrasileiro [Araucaria angustifolia (Bertol.) Kuntze], mata de sucessão secundária e silvicultura de Pinus spp. De cada uma das formações vegetais foram escolhidas duas áreas, totalizando seis áreas de amostragem. Estas áreas são caracterizadas de acordo com BAASKE (2001) como (1) duas áreas primárias (PRI1, 50¹1'37'S-29²9'22”O, $930 \mathrm{~m}$; PRI2, 50¹0'13"S-2928'41”O, 910m) de Floresta Ombrófila Mista com média concentração de A. augustifolia; (2) uma área de vegetação secundária (SEC1, 50¹0'58"S-29²9'15”O, $920 \mathrm{~m}$ ) do tipo floresta de Tibouchina sellowiana (Chan.) Cogn., formação de altitude em encosta sombreada; (3) uma área de vegetação secundária (SEC2, 5009'24"S-29²8'27'O, 915 m) do tipo floresta de T. sellowiana, sem maiores diferenciações; (4) uma área com silvicultura de Pinus taeda L. (PIN1, $50^{\circ} 11^{\prime} 24^{\prime}$ 'S-29॰29'23”O, $925 \mathrm{~m}$ ) com no máximo 30 anos de idade; (5) uma área com silvicultura de Pinus elliottii Engelm. (PIN2, 50¹1'10’'S-9²9’32”O, $870 \mathrm{~m}$ ) com no máximo 30 anos de idade.

As áreas primárias apresentam um estrato arbóreo alto com um sub-bosque de árvores finas, esparsos aglomerados de bambu e muitas fanerógamas; serrapilheira constituída de folhas de diferentes tamanhos (BAASKE, 2001). As áreas secundárias apresentam estrato arbóreo mais baixo, sem sub-bosque, caracterizadas pelas espécies de bambu e fanerógamas; a serrapilheira é heterogênea, porém constata-se a dominância de folhas de bambu. As áreas de silvicultura apresentam estrato arbóreo alto e homogêneo, com sub-bosque formado por alguns exemplares juvenis de espécies arbóreas nativas e fanerógamas; nas bordas da floresta encontramos espécies vegetais típicas de capoeira. A serrapilheira é profunda, compacta e homogênea, formada principalmente por acículas de Pinus spp.

Desenho amostral. Em cada uma das áreas foram instaladas 10 armadilhas de queda ("pitfall-traps") ao longo de dois transectos distando entre 50 e $100 \mathrm{~m}$ entre si (cada transecto com cinco armadilhas distantes $10 \mathrm{~m}$ entre si). As armadilhas de queda são compostas de copos plásticos descartáveis de $500 \mathrm{ml}$ (diâmetro $10 \mathrm{~cm}$; profundidade de $15 \mathrm{~cm}$ ) inseridos em uma guia de $\mathrm{PVC}$ de $10 \mathrm{~cm}$ de diâmetro para facilitar a remoção do material coletado e reposição do copo coletor. Cada armadilha foi coberta por um azulejo branco de $20 \times 20 \mathrm{~cm}$ disposto cerca de $10 \mathrm{~cm}$ acima da borda do copo coletor para evitar a entrada de água da chuva. Como líquido conservante foi utilizada formalina a $4 \%$ com adição de algumas gotas de detergente

Tab. I. Datas amostrais e número de Bothriurus signatus Pocock, 1893 coletados no período de 22.IX.2000 a 18. V.2002 em seis áreas no CPCN Pró-Mata, São Francisco de Paula, RS, Brasil (PRI, floresta primária; SEC, floresta secundária; PIN, silvicultura de Pinus spp.).

\begin{tabular}{|c|c|c|c|c|c|c|c|}
\hline Áreas/datas & PRI1 & PRI2 & SEC1 & SEC2 & PIN1 & PIN2 & Total \\
\hline 25.X.2000 & 3 & 3 & 0 & 0 & 1 & 0 & 7 \\
\hline 23.XI.2000 & 6 & 4 & 0 & 0 & 6 & 6 & 22 \\
\hline 02.I.2001 & 14 & 10 & 1 & 1 & 9 & 3 & 38 \\
\hline 02.II.2001 & 3 & 0 & 0 & 0 & 2 & 4 & 9 \\
\hline 03.III.2001 & 1 & 1 & 0 & 1 & 3 & 2 & 8 \\
\hline 07.IV.2001 & 0 & 2 & 0 & 0 & 1 & 1 & 4 \\
\hline 01.V.2001 & 0 & 0 & 0 & 0 & 0 & 1 & 1 \\
\hline 02.VI.2001 & 1 & 0 & 0 & 0 & 2 & 0 & 3 \\
\hline 07.VII.2001 & 0 & 0 & 0 & 0 & 0 & 0 & 0 \\
\hline 09.VIII.2001 & 0 & 1 & 0 & 0 & 2 & 0 & 3 \\
\hline 14.IX.2001 & 4 & 1 & 0 & 0 & 3 & 0 & 8 \\
\hline 11.X.2001 & 1 & 3 & 0 & 0 & 0 & 3 & 7 \\
\hline 13.XI.2001 & 8 & 7 & 0 & 1 & 2 & 5 & 23 \\
\hline 15.XII.2001 & 0 & 7 & 0 & 0 & 1 & 1 & 9 \\
\hline 02.II.2002 & 3 & 3 & 0 & 1 & 3 & 3 & 13 \\
\hline 08.III.2002 & 0 & 1 & 0 & 0 & 2 & 1 & 4 \\
\hline 14.IV.2002 & 1 & 3 & 0 & 1 & 0 & 0 & 5 \\
\hline 18.V.2002 & 0 & 0 & 1 & 0 & 0 & 0 & 1 \\
\hline Total & 45 & 46 & 2 & 5 & 37 & 30 & 165 \\
\hline
\end{tabular}


para neutralizar a tensão superficial. O líquido coletor foi substituído a cada amostragem.

O período de amostragem foi de 22 de setembro de 2000 a 18 de maio de 2002, totalizando 602 dias, o que possibilitou a coleta em dois verões e um inverno. A retirada das amostras foi realizada ao longo de 18 datas amostrais (Tab. I). Não foi estabelecido um número padrão de dias para cada período amostral. Por este motivo, a atividade sazonal dos escorpiões foi medida como uma fração do total de indivíduos coletados em relação ao número de dias em cada período amostral, calculada a média de indivíduos coletados por dia em cada período amostral.

Comparação entre áreas. Para a comparação das áreas, dados de abundância dos escorpiões foram avaliados através da análise do erro padrão da abundância absoluta das 10 armadilhas instaladas em cada uma das áreas.

Tamanho corporal dos escorpiões. Para estabelecer a relação de tamanho dos escorpiões foram mensurados o comprimento total e a medida da carapaça de todos os indivíduos (incluindo jovens) com auxílio de microscópio estereoscópico com ocular milimetrada. Para comparação do tamanho de machos e fêmeas foi utilizada a medida do comprimento da carapaça e o teste de Mann-Whitney $(\mathrm{p}<0,05)$ para dados não paramétricos. A medida comparativa entre as diferentes fisionomias foi testada comparando-se a média do comprimento das carapaças dos escorpiões machos em cada uma das três formações vegetais através de ANOVA (JANDEL, 1995). Fêmeas foram dissecadas e examinadas para verificar presença ou ausência de embriões e eventual confirmação do período reprodutivo da espécie.

Material testemunho. Exemplares estão depositados nas coleções do Museu de Ciências Naturais da Fundação Zoobotânica do Rio Grande do Sul (R. Ott) e do Museu de Ciências e Tecnologia da Pontifícia Universidade Católica do Rio Grande do Sul (A. A. Lise), ambas em Porto Alegre, RS.

\section{RESULTADOS}

O levantamento de escorpiões em serrapilheira nos três tipos de formações vegetais (florestas primárias, florestas secundárias e silviculturas) registrou apenas Bothriurus signatus Pocock, 1893.

Abundância e sazonalidade. Foram registrados 165 escorpiões, representados por 92 machos (56\%), 38 fêmeas $(23 \%)$ e 35 jovens (21\%). Os números totais coletados em cada uma das formações vegetais (Tab. I) indicam que o total de indivíduos foi maior nas áreas primárias (91 indivíduos) e silviculturas (67) e menor nas áreas secundárias (7). O número de escorpiões mostrou-se significativamente menor nas áreas de vegetação secundária, mas não apresentou diferença estatística entre as áreas de floresta primária e silvicultura (Fig. 1).

A distribuição dos indivíduos capturados ao longo do ano indica predominância durante os meses de primavera e verão, com números máximos registrados ao longo de dezembro de 2000 e outubro-novembro de 2001 (Fig. 2). A atividade desta espécie mostra clara sazonalidade, registrando nos meses de abril a junho os menores índices de atividade (Figs. 3, 4). Machos e fềmeas foram registrados na mesma armadilha nas seguintes datas amostrais: 25.X.2000, 22.XI.2000, 14.IX.2001, 11.X.2001 e 13.XI.2001. Não ocorreram fêmeas fertilizadas (grávidas) com presença de embriões.

Variação de tamanho corporal dos escorpiões. O comprimento total dos indivíduos variou de 0,95 a 3,49 $\mathrm{cm}$, com o comprimento da carapaça entre 1,23 e 4,52 $\mathrm{mm}$ (Fig. 5). Para machos, a variação ficou entre 2,43 e $3,49 \mathrm{~cm}$ para o comprimento total e 3,15 a $4,52 \mathrm{~mm}$ para o comprimento da carapaça. Para as fêmeas as medidas foram, respectivamente: 2,33 a 3,17 $\mathrm{cm}$ e 3,01 a 4,11 mm. A diferença do tamanho da carapaça entre machos e fêmeas mostrou-se significativa $(\mathrm{T}=1882,5 ; \mathrm{P}=0,002)$, sendo a média de tamanho registrada nos machos de $3,77 \mathrm{~mm}$ e nas fêmeas $3,57 \mathrm{~mm}$. Não houve diferença significativa nas médias de tamanho da carapaça dos escorpiões machos entre as formações vegetais (PRI $=3,75 \mathrm{~mm}$; $\mathrm{SEC}=3,49$ $\mathrm{mm} ; \mathrm{PIN}=3,80 \mathrm{~mm} ; \mathrm{H}=2,170 ; \mathrm{P}=0,338)$.

\section{DISCUSSÃO}

O registro de apenas uma espécie de escorpião na serrapilheira ao longo dos 602 dias revela que o ambiente apresenta baixa riqueza. Certamente um dos fatores limitantes, preponderantes no local, é o clima extremo da região, mais frio (em função da altitude) do que em regiões vizinhas como, por exemplo, áreas do Bioma da Mata Atlântica ou do Bioma Pampa, o que corrobora o proposto por Polis (1990). Entretanto considera-se que apenas a serrapilheira foi amostrada, sem coletas em locais crípticos como pedras, troncos ou outros abrigos que poderiam ser utilizados por este grupo. Embora a região se localize dentro do limite sul da Mata Atlântica, sua altitude em torno de $1000 \mathrm{~m}$ gera temperaturas médias anuais mais baixas do que em regiões próximas, o que pode ser considerado um fator limitante para a diversidade de escorpiões.

Embora escorpiões apresentem taxocenoses regionais de ampla distribuição, algumas espécies e até mesmo espécies de outras ordens de aracnídeos como opiliões são aparentemente restritas a tipos específicos de habitats dentro de ecossistemas locais (AcosTA, 1995a,b, 2002; Acosta \& Ferradés, 1996; Acosta \& MAury, 1998). A Floresta com Araucária constitui um ecossistema único e com características vegetais e climáticas diferenciadas em relação a outras formações vegetais no Rio Grande do Sul. Desta maneira é possível que a espécie encontrada apresente distribuição restrita a este ecossistema ou apenas a ecossistemas adjacentes à Floresta Ombrófila Mista como a Mata Atlântica, onde já foi registrada.

A quantidade total de indivíduos coletada a partir do esforço empregado é aparentemente baixa para um período de coleta contínua de 20 meses. Todavia o número de escorpiões capturados, considerando coletas representadas 


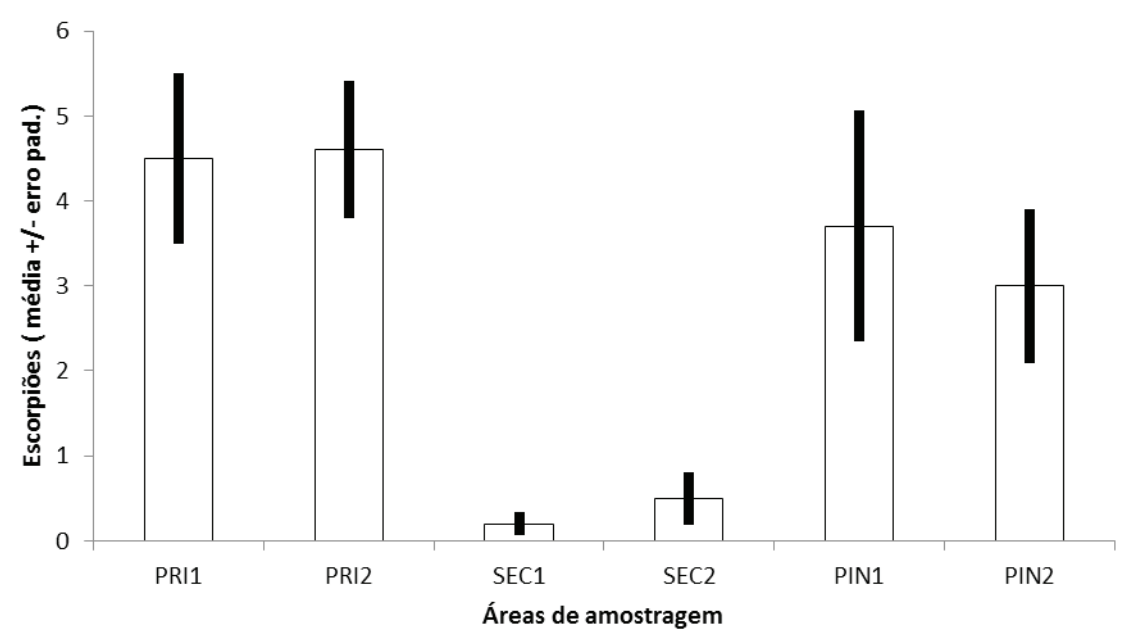

Fig.1. Abundância de Bothriurus signatus Pocock, 1893 e erro padrão das médias (barras escuras) registradas em cada uma das 10 armadilhas de queda instaladas em cada área amostral, de 22.IX.2000 a 18.V.2002, CPCN Pró-Mata, São Francisco de Paula, RS, Brasil.

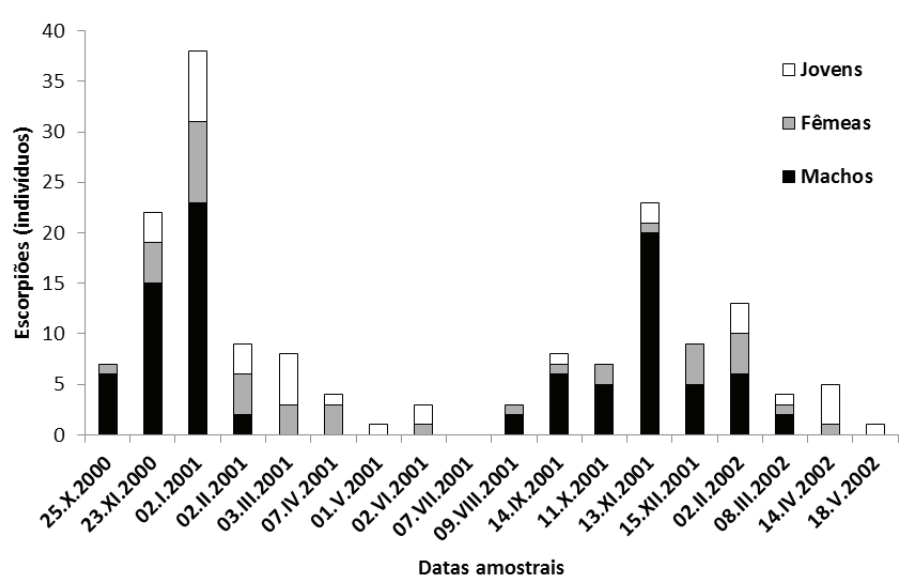

Fig. 2. Abundância de Bothriurus signatus Pocock, 1893 nas respectivas datas amostrais, CPCN Pró-Mata, São Francisco de Paula, RS, Brasil.

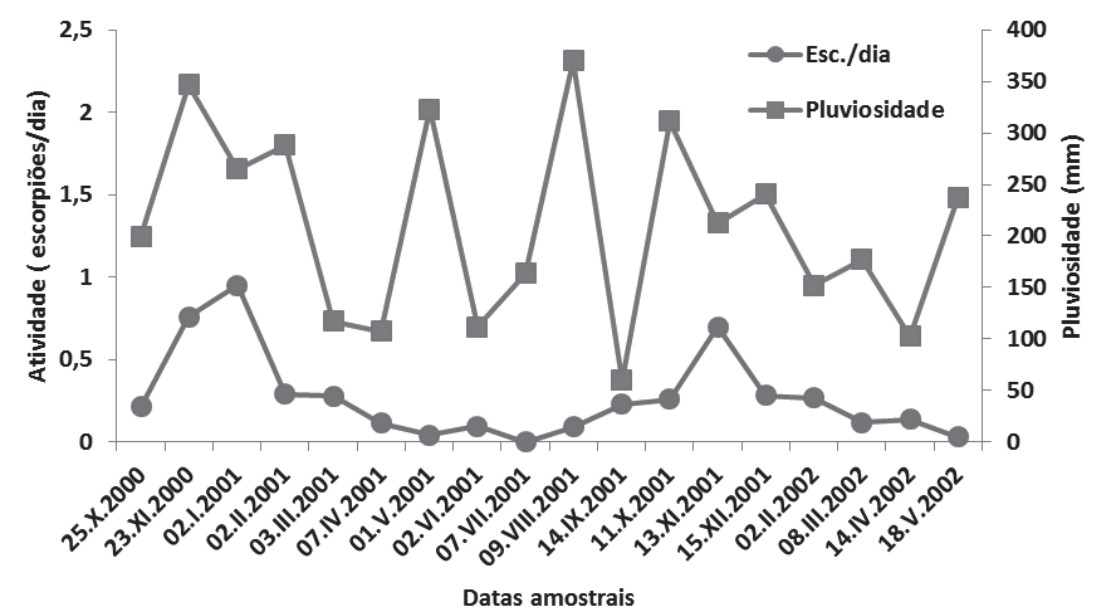

Fig. 3. Atividade de Bothriurus signatus Pocock, 1893 (médias de escorpiões/dia) e médias mensais de pluviosidade entre as datas amostrais de 22.IX.2000 a 18.V.2002 no CPCN Pró-Mata em São Francisco de Paula, RS, Brasil. 


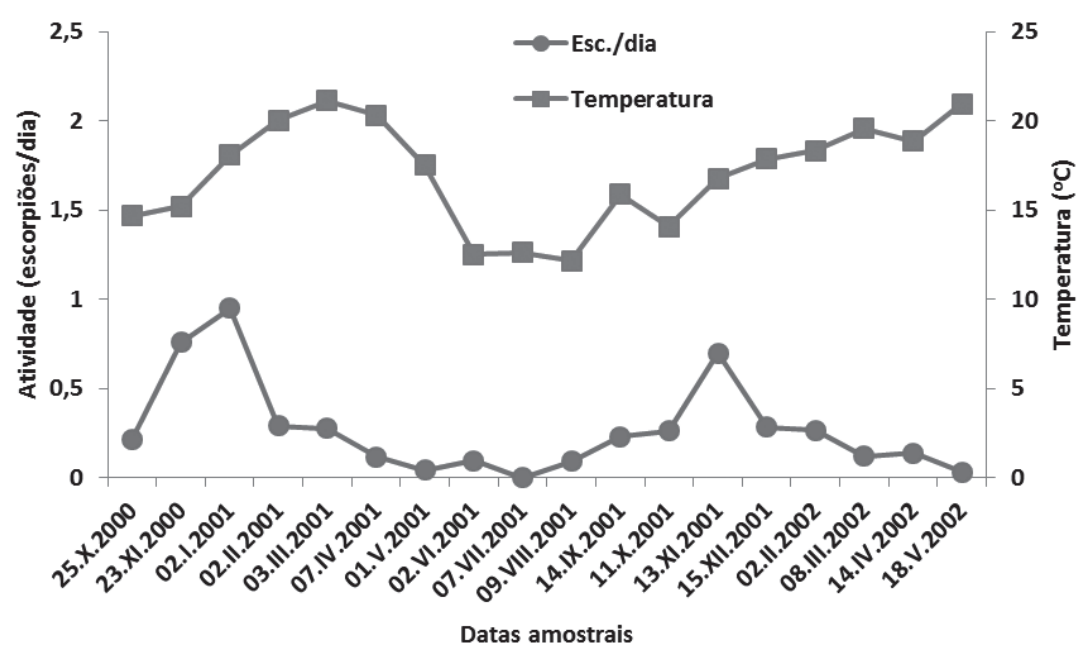

Fig. 4. Atividade de Bothriurus signatus Pocock, 1893 (médias de escorpiões/dia) e médias mensais de temperatura entre as datas amostrais de 22.IX.2000 a 18.V.2002 no CPCN Pró-Mata em São Francisco de Paula, RS, Brasil.

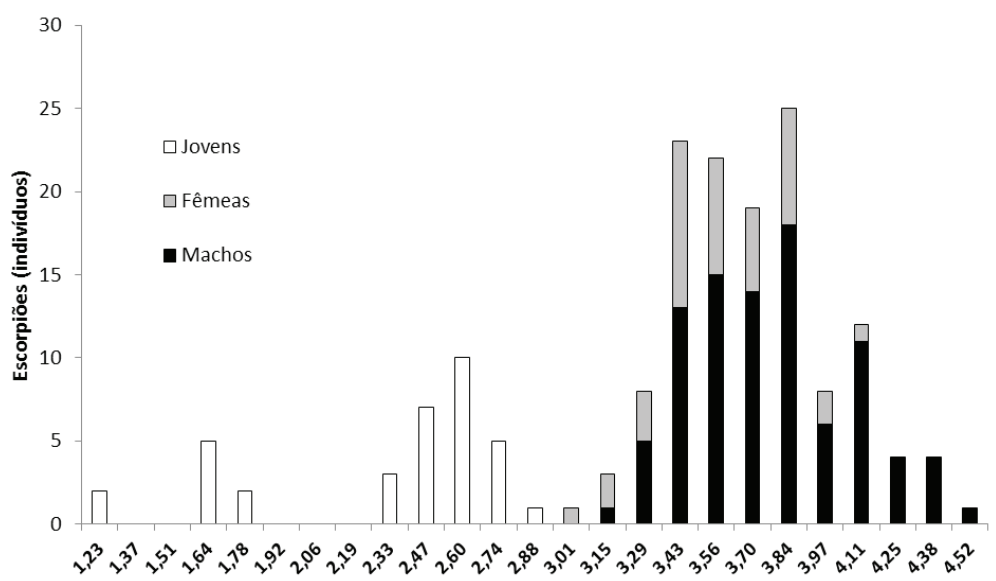

Fig. 5. Comprimento médio da carapaça de Bothriurus signatus Pocock, 1893 registrados de 22.IX.2000 a 18.V.2002 no CPCN Pró-Mata em São Francisco de Paula, RS, Brasil.

por apenas uma espécie, parece estar próximo de registros de autores como Corey \& Stout (1990), inclusive para as proporções de machos, fêmeas e jovens obtidos em dunas arenosas na Flórida (Estados Unidos). Para regiões desérticas, o método de "pitfall" parece ser mais eficiente, tanto em função da abundância quanto da riqueza (Sheнав et al., 2011). Porém, devemos considerar que a metodologia de armadilhas de queda está intimamente ligada a características de maior ou menor atividade de cada espécie e estas, condicionadas a diferentes fatores como, por exemplo, o clima de cada região e o período de amostragem, não refletindo necessariamente a abundância absoluta ou densidade populacional dos animais de uma área. ToSCANO-GAEDA (2002), ao utilizar cerca de um terço do esforço amostral com 22 armadilhas de menor tamanho (7,5 cm de diâmetro) durante dois anos de amostragens ininterruptas, coletou 79 escorpiões no Uruguai, contudo, de quatro espécies distintas, sendo uma delas introduzida. ARAúJo et al. (2010), também com metodologia similar e o uso de 36 armadilhas ao longo de dois anos, coletaram
104 indivíduos de quatro espécies distintas em uma área de Caatinga na Paraíba.

Assim como neste trabalho, um maior número de machos para espécies de Bothriuridae também foi registrado por Araúso et al. (2010) e Yamaguchi \& Pinto-DA-Rocha (2006). Machos tendem a ser mais ativos do que fêmeas nos períodos reprodutivos (WARBURG \& Polis, 1990), refletindo em maior número de exemplares capturados. Todavia o maior número de machos registrados, em função de sua maior atividade, não implica, necessariamente, em maior número de indivíduos deste sexo na população amostrada.

De acordo com a diferença de abundância entre as áreas, o baixo número observado na floresta secundária poderia levar à suposição de que a espécie seja bastante sensível às condições de qualidade ambiental. Em termos de condições de solo, as áreas estudadas são homogêneas, com solo relativamente raso e pedregoso característico de topo de morros basálticos, não existindo influência de fatores como, por exemplo, presença de corpos d'água que poderiam condicionar a presença desta espécie. 
Entretanto os maiores números registrados nas silviculturas contradizem, em parte, a suposição de que a espécie possa ser sensível à qualidade do ambiente, pelo menos no que diz respeito ao tipo de cobertura vegetal. O que chama a atenção em áreas de floresta secundária, ao contrário das áreas primárias e silviculturas, é a menor quantidade de serrapilheira. Embora os escorpiões não sejam diretamente dependentes da serrapilheira como recurso alimentar, o elevado número de escorpiões nas áreas de silvicultura pode estar relacionado à maior disponibilidade de abrigo e presas, sendo que a baixa qualidade da serrapilheira nestas áreas poderia ser compensada pela quantidade e profundidade, aumentando sua presença. COREy \& Stout (1990) registraram menos indivíduos em áreas de serrapilheira mais profunda, diferentemente do verificado neste trabalho.

Candido (1999) afirmou que os escorpiões apresentam maior atividade durante os períodos de verão; contudo, segundo WARBURG \& Polis (1990), seriam conhecidos dois tipos principais de padrões de atividade: maior atividade no período de verão ou maior no período de inverno; estes padrões diferem dos resultados encontrados neste estudo (Figs 3, 4). Bothriurus signatus apresentou maior atividade no período de primavera, que não corresponde ao período mais quente nem ao período mais frio do ano nesta região. Os resultados obtidos diferem do observado por Corey \& Stout (1990) e Costa et al. (1991), que registraram a maior atividade durante os meses de verão. Devido ao clima mais frio da Floresta com Araucária, seria esperado um atraso no início do período de maior de atividade dos escorpiões, entretanto, o que ocorre é justamente o contrário. Na área estudada, picos de atividade foram registrados ao longo dos meses de outubro/novembro, muito antes das temperaturas atingirem o seu máximo.

Segundo Warburg \& Polis (1990), uma das exceções ao padrão de maior atividade, no inverno ou no verão, seria o escorpião de áreas desérticas Smeringurus mesaensis (Stahnke, 1957), que apresenta atividade reduzida tanto no inverno como no verão, possivelmente relacionada com ocorrência de temperaturas extremas.

Os dados deste estudo não se enquadram em nenhum dos padrões acima: a maior atividade ocorreu na primavera, sem a ocorrência de outros picos sazonais, os quais aparentemente não estão relacionados somente à temperatura. A maior atividade registrada na primavera para B. signatus poderia estar relacionada à maior pluviosidade neste período, o que é normal para a região de São Francisco de Paula. Yamaguti \& Pinto-DA-Rocha (2006) encontraram padrão de atividade similar, com picos no período de primavera para Thestylus auriantiurus Yamaguti \& Pinto-da-Rocha, 2003 na Serra da Cantareira, em São Paulo. Dados de ARAúso et al. (2010) relacionaram maior atividade de escorpiões ao aumento de pluviosidade o que, em parte, corroboraria os dados obtidos para a sazonalidade de B. signatus.

É possível que, no caso dessa espécie, ocorra um efeito combinado de aumento de temperatura e aumento da pluviosidade como um gatilho para a intensificação da atividade reprodutiva, já que o número de machos cresce muito mais que o de fêmeas neste período (Fig. 2). Isso também parece estar evidenciado nos resultados ora apresentados (Fig. 3); em ambos os anos (2000 e 2001) os períodos de maior atividade da espécie são registrados nos meses imediatamente posteriores aos meses com aumento de pluviosidade e após a temperatura média ter registrado mais de $15^{\circ} \mathrm{C}$.

Segundo Yamaguti \& Pinto-da-Rocha (2006), o registro de machos e fêmeas na mesma armadilha poderia estar relacionado com a "dança" de acasalamento, quando o casal de escorpiões busca um local para depósito do espermatóforo. Para machos e fêmeas de B. signatus encontrados na mesma armadilha e no mesmo período, foram registrados predominantemente para a primavera, reforçando a possibilidade de que este seja efetivamente $o$ período reprodutivo para esta espécie. No exame das fêmeas coletadas neste trabalho, nenhuma apresentou embriões formados ou reconhecíveis, dificultando a utilização deste dado para confirmação do período reprodutivo. Segundo Polis (1990) apud Yamaguti \& Pinto-DA-Rocha (2006), de acordo com as estratégias de caça dos escorpiões do tipo "senta-e-espera", os mesmos seriam mais ativos para busca de parceiros do que para busca de presas e, portanto, seria possível concluir que fêmeas "grávidas" (já fertilizadas) são menos propensas à captura por armadilhas de queda do que fêmeas em busca de parceiros.

De acordo com Polis \& Sissom (1990), na maioria das espécies de escorpiões as fêmeas são menores do que os machos; contudo, segundo os mesmos autores, em algumas espécies as fêmeas podem ser maiores dos que os machos, como registrado neste trabalho.

Análises de variação no tamanho de escorpiões e outros aracnídeos entre diferentes formações vegetais, considerando-se variações interespecíficas, não são conhecidas na literatura. Os resultados encontrados neste estudo não indicaram diferenças significativas entre o tamanho dos escorpiões machos amostrados nas áreas de floresta primária, secundária e silvicultura. Todavia considera-se necessário o aprofundamento deste tipo de estudo uma vez que a qualidade do ambiente está relacionada com a qualidade e disponibilidade recursos para escorpiões (McCormick \& Polis, 1990).

Agradecimentos. Ao Dr. Camillo Matoni (Universidad Nacional de Córdoba, Argentina) pela confirmação da identificação da espécie. À PUCRS pela disponibilização da área para a realização deste estudo. À CAPES pelo apoio financeiro. Aos revisores anônimos pelas sugestões apresentadas. Este trabalho é parte integrante da Tese de Doutorado em Zoologia (PUCRS) do primeiro autor.

\section{REFERÊNCIAS BIBLIOGRÁFICAS}

Acosta, L. E. 1995a. The Scorpions of Western Chaco. I. Diversity and distributional patterns. Biogeographica 71(2):49-59. . 1995b. The Scorpions of Western Chaco. I. Community survey in the Llanos district. Biogeographica 71(4):187-196. 2002. Patrones zoogeográficos de los opiliones argentinos 
(Arachnida: Opiliones). Revista Ibérica de Aracnología 6:69-84.

Acosta, L. E. \& Maury, E. A. 1998. Scorpiones. In: Morrone, J. J. \& Coscarón, S. eds. Biodiversidad de Artrópodos Argentinos. La Plata, Ediciones SUR. p. 545-559.

Acosta, L. E. \& Rosso de Ferradás, B. 1996. Arácnidos de la Provincia de Córdoba, Argentina. Bulletin of the British Arachnological Society 10(1):23-28.

Araújo, C. S.; Candido, D. M.; Araújo, H. F. P. de; Dias, S. C. \& VAsconcellos, A. 2010. Seasonal variations in scorpion activities (Arachnida: Scorpiones) in an area of Caatinga vegetation in northeastern Brazil. Zoologia 27(3):372-376

Batske, R. 2001. Vegetationskartierung: des forschunsgebiets PróMata, Rio Grande do Sul, Brasilien, unter verwendung von CIR - luftbidern. CD-ROM.

BACKES, A. 2009. Distribuição geográfica atual da floresta com araucária: condicionamento climático. In: FonseCA, C. R. ed. Floresta com araucária: ecologia, conservação e desenvolvimento sustentável. Ribeirão Preto, Holos. p. 39-44.

Bertoletti, J. J. \& Teixeira, M. B. 1995. Centro de Pesquisa e conservação da Natureza Pró-Mata. Termo de referência. Divulgação do Museu de Ciência e Tecnologia - UBEA/PUCRS 2:1-47.

Bonaldo, A. B.; Carvalho, L. S.; Pinto-Da-Rocha, R.; Tourinho, A.; Miglio, L. T.; Candiani, D. F.; Lo-Man-Hung, N. F.; Abrahim, N.; Rodrigues, B. V. B.; Brescovit, A. D.; Saturnino, R. F.; Bastos, N. C.; Dias, S. C.; Silva, B. J. F.; Pereira-Filho, J. M. B.; Rheims C. A.; Lucas, S. M.; Polotow, D.; Ruiz, G. R. S. \& Indicatti, R. P. 2009. Inventário e história natural dos aracnídeos da Floresta Nacional de Caxiuanã. In: LisBoA, P. L. B. org. Caxiuanã: desafios para a conservação de uma Floresta Nacional na Amazônia. Belém, Museu Paraense Emílio Goeldi. p. 577-621.

BRAZIL, T. K. \& PoRTO, T. J. 2010. Os escorpiões. Salvador, EDUFBA. 84p.

Candido, D. M. 1999. Escorpiões. In: Brandão, C. R. F. \& CAncello, E. M. org. Biodiversidade do Estado de São Paulo. São Paulo, Fapesp. v. 5, p. 23-24

Corey, D. T. \& Stout, F. J. 1990. Ground surface arachnids in sandhill communities of Florida. Journal of Arachnology 18:167-172.

Costa, F. G.; Pérez-Miles, F.; Gudynas, E.; Prandi, L. \& Capocasale, R. M. 1991. Ecologia de los Aracnidos criptozoicos, exceto acaros, de
Sierra de Las Animas (Uruguay). Ordenes e familias. Aracnologia 13(15):1-41.

Dias, S. C.; Candido, D. M. \& Brescovit, A. D. 2006. Scorpions from Mata do Buraquinho, João Pessoa, Paraíba, Brazil, with ecological notes on a population of Ananteris mauryi Lourenço (Scorpiones, Buthidae). Revista Brasileira de Zoologia 23(3):707-710.

JANDEL, C. 1995. SigmaStat. Versão 2.0. Jandel Corporation, USA.

KöPpen, W. 1948. Climatologia. Cidade do México: Fondo de Cultura. p. 152-192.

McCormick, S. J. \& Polis, G. A. 1990. Prey, Predators, and Parasites. In: Polis, G. A. ed. The Biology of Scorpions. Standford, Standfort University Press, p. 294-320.

Mota, F. S. \& Agendes, M. O. O. 1986. Clima e Agricultura no Brasil. Porto Alegre, Sagra. 151p.

Polis, G. A. 1990. Ecology. In: Polis G. A. ed. The Biology of Scorpions. Standford, Standfort University Press, p. 247-293.

Polis, G. A. \& Sissom, W. D. 1990. Life History. In: Polis, G. A. ed. The Biology of Scorpions. Standford, Standford University Press, p. 161-223.

PRENDINI, L. 2005. Scorpion diversity and distribution in Southern Africa: Pattern and process. In: Huber, B. A.; Sinclair, B. J. \& Lampe, K. H. eds. African Biodiversity: Molecules, Organisms, Ecosystems. Proceedings of the $5^{\text {th }}$ International Symposium on Tropical Biology, Museum Alexander Koenig, Bonn. Springer Verlag, New York. p. 25-68.

SHeHAB, A. H.; AmR, Z. S. \& LindSELl, J. A. 2011. Ecology and biology of scorpions in Palmyra, Syria. Turkish Journal of Zoology 35(3):333341.

TosCANo-GadeA, C. A. 2002. Fenología y distribución de la escorpiofauna del Cerro de Montevideo, Uruguay: un studio de dos años con trampas de caída. Revista Ibérica de Aracnología 31(5):77-82.

Warburg, M. R. \& Polis, G. A. 1990. Behaviour Responses, Rhythms, and Activity patterns. In: Polis, G. A. ed. The Biology of Scorpions. Standford, Standford University Press, p. 224-246.

Yamaguti, H. Y. \& Pinto-DA-Rocha, R. 2006. Ecology of Thestylus aurantiurus of the Parque Estadual da Serra da Cantareira, São Paulo, Brazil (Scorpiones, Bothriuridae). The Journal of Arachnology 34:214-220. 